\title{
Pneumo-pyopericardium Mimicking an Acute Myocardial Infarction. A Rare Complication of an Incarcerated Hiatus Hernia and Gastro-pericardial Fistula
}

\author{
Ciprian Blendea, Adriana Crăciun, Crina Simon, Sebastian Condrea \\ Center of Advanced Research in Multimodality Cardiac Imaging, Cardio Med Medical Center, Tîrgu Mureș, Romania
}

\section{ABSTRACT}

Pyopericardium is an acquired disease associated with very high complication rates. Pyopericardium is a very rare form of pneumopericardium. The nontraumatic causes of pneumopericardium include gastro-pericardial fistula that can occur in refractory gastric ulcers, stomach or esophageal neoplasia, and in surgical interventions of the stomach, such as the Niessen fundoplication procedure. The case of life-threatening pneumopericardium associated with gastro-pericardial fistula resulting from the incarceration of a giant hiatal hernia is reported.

Keywords: penumopericardium, pyopericardium, gastro-pericardial fistula, hiatal hernia

\section{ARTICLE HISTORY}

Received: 14 February, 2016

Accepted: 20 July, 2016

\section{CORRESPONDENCE}

Adriana Crăciun

Str. 22 Decembrie 1989 Nr. 76

540124 Tîrgu Mureș, Romania

Tel: +40 265217333

E-mail: christmas_ada_47@yahoo.com

\section{INTRODUCTION}

Pyopericardium is an acquired disease associated with very high complication rates. Pyopericardium is a very rare form of pneumopericardium. Pneumopericardium is most frequently caused by traumatic injuries, but may have a nontraumatic origin. The latter may occur as a result of a benign or malignant gastric ulcer, perforating into the pericardial sack, and usually, in such cases, there is a hiatal hernia causing thoracic displacement of the stomach., ${ }^{1,2}$ This disorder could result as a complication of a gastro-pericardial fistula, a very rare and severe condition consisting of an abnormal communication be- tween the stomach and the pericardium. The main clinical signs and symptoms of a gastro-pericardial fistula include sudden intense thoracic pain that radiates to the shoulder, and is associated with dyspnea. ${ }^{3}$

A case of life-threatening pneumo-pyopericardium related to gastro-pericardial fistula resulting from the incarceration of a large hiatal hernia is reported here.

\section{CASE PRESENTATION}

A 68-year-old man with no previous cardiovascular medical history presented complaining of chest pain, associated with pain in the epigastric region and syncope. An 

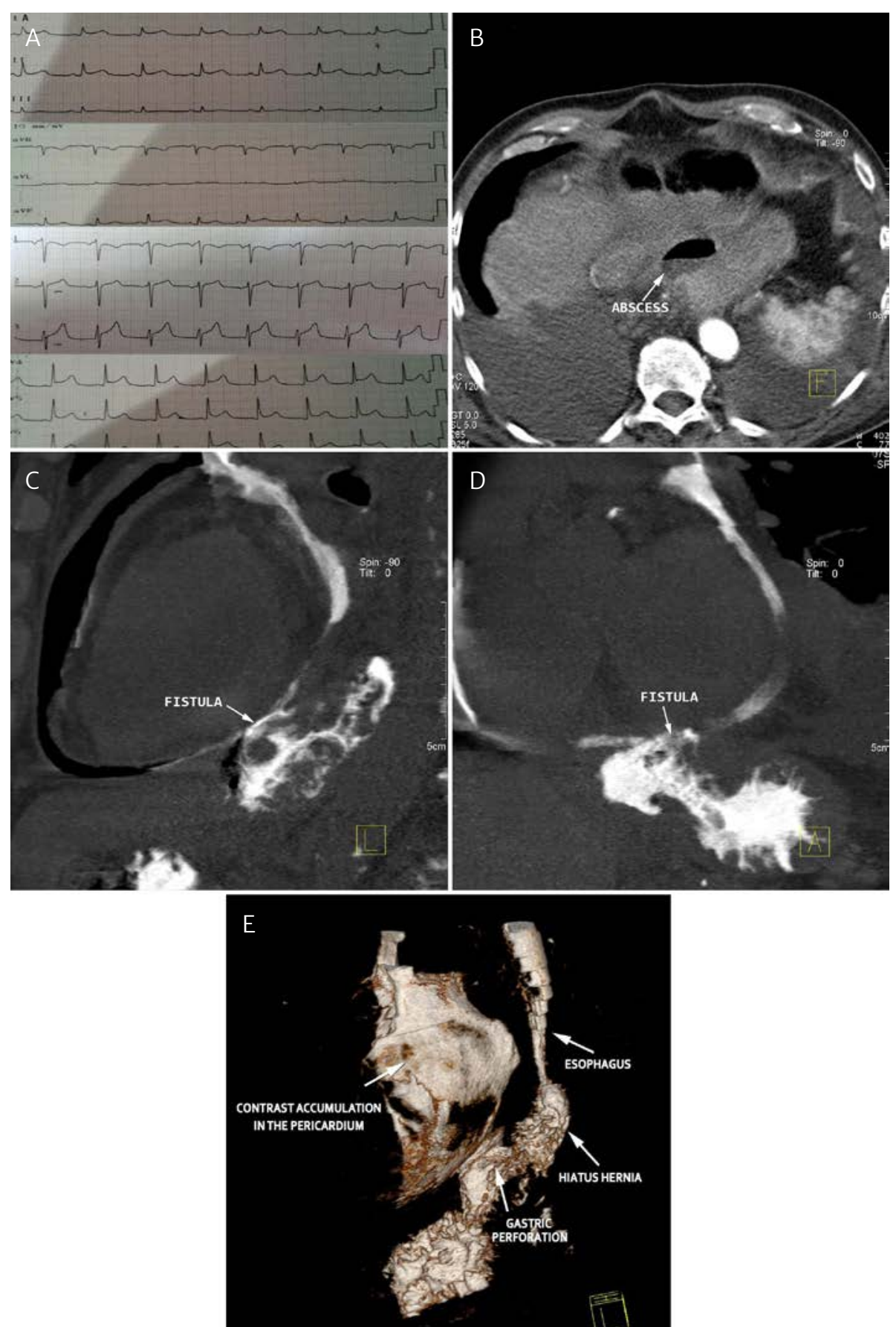

FIGURE 1. Panel A. ECG showing ST segment elevation in DI, DII, DIII, aVL, aVF, V2-V6 leads. Panel B. Thoracic CT showing an abscess associated a giant hiatus hernia, with a gastro-pericardial fistula. Panel C. Contrast enhanced thoracic CT showing air and fluid levels in the pericardial cavity, a hiatal hernia, and gastro-pericardial fistula. Panel D. Presence of contrast agent within the pericardial sack. Panel E. 3D reconstruction of the $\mathrm{CT}$ imaging, showing the gastro-pericardial fistula.

electrocardiogram showed ST segment elevation in DI, DII, DIII, aVL, aVF, V2-V6 leads (Panel A). However, the serum troponin levels were not elevated.

An emergency coronary computed tomography angiography was performed on suspicion of the presence of an acute coronary syndrome. No significant lesions in the coronary arteries were observed. A thoracic computed to- mography was performed, which revealed an abscess associated with a confinement of the stomach in a hiatus hernia, associated with a fistula between the stomach and the pericardial space (Panel B).

After the oral administration of a contrast agent, thoracic computed tomography revealed a significant amount of air in the pericardial cavity, with a maximum 
axial diameter of $11 \mathrm{~mm}$ at the level of cardiac apex, and a minimal fluid collection with multiple air inclusions, indicating a pyopericardium (Panel C). Also, the presence of a large hiatus hernia with a maximum axial diameter of $6 \mathrm{~cm}$ and an anterior gastric wall discontinuity of 12 $\mathrm{mm}$ was observed. This was associated with a very well delineated adjacent image, with both air and fluid levels and progressively increasing density (Panel C). The contrast agent was also present in the pericardial cavity (Panel D). The patient was diagnosed with pneumopericardium secondary to a gastro-pericardial fistula in a hiatus hernia. The three-dimensional computed tomography reconstruction clearly illustrated the communication between the gastric wall and the pericardium (Panel E). Surgical treatment was initiated consisting in the correction of the hiatus hernia and drainage of the pyopericardium.

The patient gave informed consent allowing publication of patient data, and the institution where the imaging data was aquired approved the publication of the case.

\section{DISCUSSION}

Pneumo-pyopericardium is a very rare disease, which is usually associated with a very poor prognosis., ${ }^{1,2}$ Patients with pneumo-pyopericardium usually present with fever and shortness of breath. ${ }^{3}$ Other symptoms include heartburn, chest pain radiating to the shoulder, and epigastralgia. In many cases, there is a severe alteration of the haemodynamic status due to the occurrence of cardiac tamponade or hypovolemic shock. ${ }^{4}$

As the disease is located at the level of the pericardium, electrocardiogram changes suggestive of acute pericarditis may be recorded, consisting in ST segment elevation in all 12 leads, and PR depression. These ECG changes, associated with chest pain, epigastralgia and syncope, in the present case, imitated an acute ST-elevation myocardial infarction (STEMI). ${ }^{5}$ In such situations the STEMI diagnosis could not be ruled out, but the lack of elevated cardiac necrosis enzymes, and also the absence of regional wall motion impairment, as assessed by $2 \mathrm{D}$ echocardiography, prevented this diagnosis. ${ }^{6}$

Nonetheless, in this case the lack of dynamic troponin level changes raised the suspicion of an uncommon presentation of another type of chest pathology. Therefore, thoracic computed tomography was undertaken and helped clarify the diagnosis by excluding the presence of any significant coronary artery stenosis, and confirming the presence of a fistula between the stomach and the pericardium.
Stomach fistulae into the pericardial sack are a very rare condition that may be encountered in several pathological conditions, such as gastric and esophageal malignant tumors, complicated peptic ulcers, and also in surgical interventions for the treatment of a hiatal hernia., ${ }^{7,8}$ The definite diagnosis of a gastro-pericardial fistula is achieved by performing several noninvasive imaging procedures, including chest X-rays that will show an enlarged mediastinum with fluid and air levels in the pericardial cavity. Besides this, a thoracic CT can be obtained for the purpose of confirming the X-ray findings, and also to establish the source of the pericardial collection. ${ }^{1,9}$

The gastro-pericardial fistula has a high death rate. ${ }^{10}$ Thus, to increase the chance of survival in a patient with pneumo-pyopericardium due to a fistula, a thorough examination, early detection, as well as emergency drainage by pericardiocentesis and surgical correction of the fistula should be performed, followed by aggressive antibiotic therapy.

The particularity of this patient was that although he had presented with symptoms that suggested a possible STEMI diagnosis, the lack of elevated myocardial necrosis biomarkers, troponin, indicated the probability of another cause of his chief complaint. The computed tomography scan revealed a giant hiatal hernia with a gastro-pericardial fistula that caused a pyopneumo-pericardium.

\section{CONCLUSION}

Incarceration of a large hiatal hernia, with the development of an abscess, and fistula formation between the gastric wall and the pericardium, resulted in a severe form of pneumo-pyopericardium. This is a very uncommon disease that may mimic an acute myocardial infarction.

\section{CONFLICT OF INTEREST}

Nothing to disclose.

\section{REFERENCES}

1. Kim WJ, Choi EJ, Oh YW, Kim KT, Kim CW. Gastropericardial fistula-induced pyopneumopericardium after esophagectomy with esophagogastrectomy. Ann Thorac Surg. 2011;91:10-11. doi: 10.1016/j.athoracsur.2010.09.082.

2. Simice $P$, Zwirewich CV. Gastropericardial fistula complicating benign gastric ulcer: case report. Can Assoc Radiol J. 2000;51:244-275.

3. Sihvo EI, Räsänen JV, Hynninen M, Rantanen TK, Salo JA. Gastropericardial fistula, purulent pericarditis, and cardiac tamponade after laparoscopic Nissen fundoplication. Ann 
Thorac Surg. 2006;81:356-358. doi:10.1016/j.athoracsur. 2004.08.048.

4. Park S, Kim JH, Lee YC, Chung JB. Gastropericardial fistula as a complication in a refractory gastric ulcer after esophagogastrostomy with gastric pull-up. Yonsei Med J. 2010;51:270-272. doi: 10.3349/ymj.2010.51.2.270.

5. Ratnayake EC, Premaratne S, Lokunarangoda N, et al. Pneumopyopericardium mimicking an inferior myocardial infarction with regional electrocardiogram changes: a case report. BMC Research Notes. 2015;8:173. doi: 10.1186/s13104-015-1143-7.

6. Thygesen K, Alpert JS, Jaffe AS, et al. Third Universal Definiton of Myocardial Infarction. Eur Heart J. 2012;33:2551-2567. doi: 10.1093/eurheartj/ehs184.
7. Murthy S, Looney J, Jaklitsch MT. Gastropericardial fistula after laparoscopic surgery for reflux disease. N Engl J Med. 2002;346:328-332. doi: 10.1056/NEJMoa010259.

8. Chinnaiyan KM, Ali MI, Gunaratnam NT. Gastric cancer presenting as gastropericardial fistula in a patient with familial adenomatous polyposis syndrome. J Clin Gastroenterol. 2004;38:298.

9. Simice P, Zwirewich CV. Gastropericardial fistula complicating benign gastric ulcer: case report. Can Assoc Radiol J. 2000;51:244-247.

10. Letoquart JP, Fasquel JL, L'Huillier JP, et al. Gastropericardial fistula. Review of the literature apropos of an original case. J Chir (Paris). 1990;127:6-12. 